PROCEEDINGS OF THE AMERICAN MATHEMATICAL SOCIETY

Volume 124, Number 9, September 1996

\title{
DERIVATIONS WITH ENGEL CONDITIONS ON MULTILINEAR POLYNOMIALS
}

\author{
PJEK-HWEE LEE AND TSIU-KWEN LEE
}

(Communicated by Ken Goodearl)

\begin{abstract}
Let $R$ be a prime algebra over a commutative ring $K$ with unity and let $f\left(X_{1}, \ldots, X_{n}\right)$ be a multilinear polynomial over $K$. Suppose that $d$ is a nonzero derivation on $R$ such that for all $r_{1}, \ldots, r_{n}$ in some nonzero ideal $I$ of $R,\left[d\left(f\left(r_{1}, \ldots, r_{n}\right)\right), f\left(r_{1}, \ldots, r_{n}\right)\right]_{k}=0$ with $k$ fixed. Then $f\left(X_{1}, \ldots, X_{n}\right)$ is central-valued on $R$ except when char $R=2$ and $R$ satisfies the standard identity $s_{4}$ in 4 variables.
\end{abstract}

Throughout this note $K$ will denote a commutative ring with unity and $R$ will denote a prime $K$-algebra with center $\mathcal{Z}$. By $d$ we always mean a nonzero derivation on $R$. For $x, y \in R$, set $[x, y]_{0}=x,[x, y]_{1}=[x, y]=x y-y x$ and, for $k>1,[x, y]_{k}=$ $\left[[x, y]_{k-1}, y\right]$.

A well-known result proved by Posner [10] states that $R$ must be commutative if $[d(x), x] \in \mathcal{Z}$ for all $x \in R$. In [7], the authors generalized Posner's theorem by showing that a Lie ideal $L$ of $R$ must be contained in $\mathcal{Z}$ if char $R \neq 2$ and $[d(x), x] \in \mathcal{Z}$ for all $x \in L$. As to the case when char $R=2$, Lanski [5] obtained the same conclusion except when $R$ satisfies the standard identity $s_{4}$ in 4 variables. On the other hand, Vukman [11] showed that $R$ is commutative if char $R \neq 2$ and $[d(x), x]_{2}=0$ for all $x \in R$, or if char $R \neq 2,3$ and $[d(x), x]_{2} \in \mathcal{Z}$ for all $x \in R$. In a recent paper [6], a full generalization of these results was proved by Lanski. He showed that a Lie ideal $L$ of $R$ is in $\mathcal{Z}$ if for some fixed $k>0,[d(x), x]_{k}=0$ for all $x \in L$, unless char $R=2$ and $R$ satisfies $s_{4}$. Note that a noncentral Lie ideal of $R$ contains all the commutators $[x, y]$ for $x, y$ in some nonzero ideal of $R$ except when char $R=2$ and $R$ satisfies $s_{4}$. It is natural to consider the situation when $[d(x), x]_{k}=0$ for all commutators $x=\left[x_{1}, x_{2}\right]$, or more generally, when $[d(x), x]_{k}=0$ for all $x=f\left(x_{1}, \ldots, x_{n}\right)$ where $f\left(X_{1}, \ldots, X_{n}\right)$ is a multilinear polynomial over $K$. In the present paper, we shall extend Lanski's theorem by imposing the condition $\left[d\left(f\left(x_{1}, \ldots, x_{n}\right)\right), f\left(x_{1}, \ldots, x_{n}\right)\right]_{k}=0$ on some nonzero ideal of $R$.

First we dispose of the simplest case when $R$ is the matrix ring $M_{m}(F)$ over a field $F$ and $d$ is an inner derivation on $R$.

Received by the editors November 4, 1994 and, in revised form, March 1, 1995.

1991 Mathematics Subject Classification. Primary 16W25; Secondary 16N60, 16R50, 16U80.

Key words and phrases. Multilinear polynomial, derivation, generalized polynomial identity.

(C)1996 American Mathematical Society 
Proposition. Let $F$ be a field and $R=M_{m}(F)$, the $m \times m$ matrix algebra over $F$. Suppose that $a \in R$ and that $f\left(X_{1}, \ldots, X_{n}\right)$ is a multilinear polynomial over $F$ such that $\left[a, f\left(x_{1}, \ldots, x_{n}\right)\right]_{k}=0$ for all $x_{i} \in R$, where $k>0$ is a fixed integer. Then either $a \in \mathcal{Z}$ or $f\left(X_{1}, \ldots, X_{n}\right)$ is central-valued on $R$ unless char $F=2$ and $m=2$.

Proof. If $m=1$, there is nothing to prove, so we assume that $m \geq 2$. We assume further that either $m \neq 2$ or char $F \neq 2$ and proceed to show that $a \in \mathcal{Z}$ if $f\left(X_{1}, \ldots, X_{n}\right)$ is not central-valued on $R$. By [8, Lemmas 2 and 9], there exists a sequence of matrices $r=\left(r_{1}, \ldots, r_{n}\right)$ in $R$ such that $f(r)=f\left(r_{1}, \ldots, r_{n}\right)=\sum_{i=1}^{m} \alpha_{i} e_{i i}$ is a noncentral diagonal matrix, say, $\alpha_{1} \neq \alpha_{2}$. Let $\varphi$ be the inner automorphism on $R$ defined by $x^{\varphi}=\left(1+e_{21}\right) x\left(1-e_{21}\right)$. Write $D=f(r)$; then $E=f\left(r^{\varphi}\right)=$ $f\left(r_{1}^{\varphi}, \ldots, r_{n}^{\varphi}\right)=D^{\varphi}=D+\left(\alpha_{1}-\alpha_{2}\right) e_{21}$. By assumption, we have $[a, E]_{k}=$ 0 , that is, $\sum_{i=0}^{k}(-1)^{i}\left(\begin{array}{c}k \\ i\end{array}\right) E^{i} a E^{k-i}=0$. Multiplying $e_{22}$ from both sides, we have $\left(\alpha_{2}-\alpha_{1}\right)^{k} e_{21} a e_{22}=0$ by using $E^{i}=D^{i}+\left(\alpha_{1}^{i}-\alpha_{2}^{i}\right) e_{21}, e_{22} E^{i}=\alpha_{2}^{i}\left(e_{22}-e_{21}\right)+\alpha_{1}^{i} e_{21}$ and $E^{i} e_{22}=\alpha_{2}^{i} e_{22}$. Thus, $e_{21} a e_{22}=0$ and so the $(1,2)$-entry $a_{12}$ of $a$ is 0 . For $s \neq t$, let $\sigma$ be a permutation in the symmetric group $S_{m}$ such that $\sigma(1)=s$ and $\sigma(2)=t$. Let $\psi$ be the automorphism on $R$ defined by $x^{\psi}=\left(\sum_{i, j} \xi_{i j} e_{i j}\right)^{\psi}=$ $\sum_{i, j} \xi_{i j} e_{\sigma(i), \sigma(j)}$. Then $f\left(r^{\psi}\right)=D^{\psi}$ is a diagonal matrix with $(s, s)-$ and $(t, t)-$ entries distinct. Replacing $D$ by $D^{\psi}$ and proceeding as before, we have $a_{s t}=0$ for $s \neq t$. In other words, $a$ is a diagonal matrix. For any $F$-automorphism $\theta$ of $R, a^{\theta}$ enjoys the same property as $a$ does, namely, $\left[a^{\theta}, f\left(x_{1}, \ldots, x_{n}\right)\right]_{k}=0$ for all $x_{i} \in R$. Hence, $a^{\theta}$ must be diagonal. Write $a=\sum_{i=1}^{m} a_{i i} e_{i i}$; then for $s \neq t$, we have $\left(1+e_{t s}\right) a\left(1-e_{t s}\right)=\sum_{i=1}^{m} a_{i i} e_{i i}+\left(a_{s s}-a_{t t}\right) e_{t s}$ diagonal. Hence, $a_{s s}=a_{t t}$ and so $a$ is a scalar matrix.

We are now ready to prove the main

Theorem. Let $R$ be a prime $K$-algebra and $f\left(X_{1}, \ldots, X_{n}\right)$ a multilinear polynomial over $K$. Suppose that $d$ is a nonzero derivation on $R$ such that

$$
\left[d\left(f\left(x_{1}, \ldots, x_{n}\right)\right), f\left(x_{1}, \ldots, x_{n}\right)\right]_{k}=0
$$

for all $x_{i}$ in some nonzero ideal $I$ of $R$, where $k>0$ is fixed. Then $f\left(X_{1}, \ldots, X_{n}\right)$ is central-valued on $R$ except when char $R=2$ and $R$ satisfies $s_{4}$.

Proof. Assume first that $d$ is $Q$-inner, that is, $d(x)=[a, x]$ for all $x \in R$, where $a$ is a noncentral element in the symmetric quotient ring $Q$ of $R$ [4]. Then

$$
\left[a, f\left(x_{1}, \ldots, x_{n}\right)\right]_{k+1}=0 \quad \text { for all } x_{i} \in I .
$$

By a theorem due to Chuang [1, Theorem 2], this generalized polynomial identity $\left[a, f\left(X_{1}, \ldots, X_{n}\right)\right]_{k+1}$ is also satisfied by $Q$. In case the center $C$ of $Q$ is infinite, we 
have $\left[a, f\left(x_{1}, \ldots, x_{n}\right)\right]_{k+1}=0$ for all $x_{i} \in Q \underset{C}{\otimes} \bar{C}$ where $\bar{C}$ is the algebraic closure of $C$. Since both $Q$ and $Q \underset{C}{\otimes} \bar{C}$ are prime and centrally closed [2, Theorems 2.5 and 3.5], we may replace $R$ by $Q$ or $Q \underset{C}{\otimes} \bar{C}$ according as $C$ is finite or infinite. Thus we may assume that $R$ is centrally closed over $C$ which is either finite or algebraically closed and $\left[a, f\left(x_{1}, \ldots, x_{n}\right)\right]_{k+1}=0$ for all $x_{i} \in R$.

By Martindale's theorem [9], $R$ is then a primitive ring having a nonzero socle $H$ with $C$ as the associated division ring. In light of Jacobson's theorem [3, p. 75], $R$ is isomorphic to a dense ring of linear transformations on some vector space $V$ over $C$, and $H$ consists of the linear transformations in $R$ of finite rank. Assume first that $V$ is finite-dimensional over $C$. Then the density of $R$ on ${ }_{C} V$ implies that $R \cong M_{m}(C)$ where $m=\operatorname{dim}_{C} V$. By the preceding proposition, $f\left(X_{1}, \ldots, X_{n}\right)$ is central-valued on $R$ unless char $R=2$ and $m=2$. Assume next that $V$ is infinite-dimensional over $C$. For any $e=e^{2} \in H$, we have $e R e \cong M_{m}(C)$ with $m=\operatorname{dim}_{C} V e$. Since $R$ satisfies $e\left[a, f\left(e x_{1} e, \ldots, e x_{n} e\right)\right]_{k+1} e=0, e \operatorname{Re}$ satisfies $\left[e a e, f\left(x_{1}, \ldots, x_{n}\right)\right]_{k+1}=0$. As we have seen above, eae must be central in $e$ Re if $m \geq 3$ and if $f\left(X_{1}, \ldots, X_{n}\right)$ is not central-valued on $e R e$. Since $a \notin C, a$ does not centralize the nonzero ideal $H$ of $R$, so $a h_{0} \neq h_{0} a$ for some $h_{0} \in H$. Also, $f\left(X_{1}, \ldots, X_{n}\right)$ is not central-valued on $H$, for otherwise $R$ would satisfy the polynomial identity $\left[f\left(X_{1}, \ldots, X_{n}\right), X_{n+1}\right]$ contrary to the infinite-dimensionality of ${ }_{C} V$. So, $\left[f\left(h_{1}, \ldots, h_{n}\right), h_{n+1}\right] \neq 0$ for some $h_{1}, \ldots, h_{n}, h_{n+1} \in H$. By Litoff's theorem [5, p. 280], there is an idempotent $e \in H$ so that $h_{0}, h_{0} a, a h_{0}, h_{1}, \ldots, h_{n}, h_{n+1}$ are all in $e R e$. Since $\operatorname{dim}_{C} V$ is infinite, we may choose $e$ so that $m=\operatorname{dim}_{C} V e \geq 3$. Then eae is central in $e$ Re because $f\left(X_{1}, \ldots, X_{n}\right)$ is not central-valued on $e R e$. Thus

$$
a h_{0}=e a h_{0}=e a e h_{0}=h_{0} e a e=h_{0} a e=h_{0} a,
$$

a contradiction. Hence $f\left(X_{1}, \ldots, X_{n}\right)$ must be central-valued on $R$ except when char $R=2$ and $R$ satisfies $s_{4}$.

Now assume that $d$ is not $Q$-inner. Recall that $d$ can be extended uniquely to a derivation $\bar{d}$ on $Q$ [4]. We denote by $f^{d}\left(X_{1}, \ldots, X_{n}\right)$ the polynomial obtained from $f\left(X_{1}, \ldots, X_{n}\right)$ by replacing each coefficient $\alpha$ with $\bar{d}(\alpha \cdot 1)$. Since

$$
\begin{aligned}
& {\left[d\left(f\left(x_{1}, \ldots, x_{n}\right)\right), f\left(x_{1}, \ldots, x_{n}\right)\right]_{k}} \\
& =\left[f^{d}\left(x_{1}, \ldots, x_{n}\right)+f\left(d\left(x_{1}\right), x_{2}, \ldots, x_{n}\right)\right. \\
& \left.\quad+\cdots+f\left(x_{1}, \ldots, x_{n-1}, d\left(x_{n}\right)\right), f\left(x_{1}, \ldots, x_{n}\right)\right]_{k}=0
\end{aligned}
$$

we have

$$
\begin{aligned}
& {\left[f^{d}\left(x_{1}, \ldots, x_{n}\right)+f\left(y_{1}, x_{2}, \ldots, x_{n}\right)\right.} \\
& \left.\quad+\cdots+f\left(x_{1}, \ldots, x_{n-1}, y_{n}\right), f\left(x_{1}, \ldots, x_{n}\right)\right]_{k}=0
\end{aligned}
$$

for all $x_{1}, \ldots, x_{n}, y_{1}, \ldots, y_{n} \in R$ by Kharchenko's theorem [4]. In particular, $\left[f^{d}\left(x_{1}, \ldots, x_{n}\right), f\left(x_{1}, \ldots, x_{n}\right)\right]_{k}=0$ and $\left[f\left(y_{1}, x_{2}, \ldots, x_{n}\right), f\left(x_{1}, \ldots, x_{n}\right)\right]_{k}=0$ for all $x_{1}, \ldots, x_{n}, y_{1} \in R$. Applying $d$ to $\left[f\left(y_{1}, x_{2}, \ldots, x_{n}\right), f\left(x_{1}, \ldots, x_{n}\right)\right]_{2 k}=0$, 
we have

$$
\begin{aligned}
& {\left[f^{d}\left(y_{1}, x_{2}, \ldots, x_{n}\right)+f\left(d\left(y_{1}\right), x_{2}, \ldots, x_{n}\right)\right.} \\
& \left.\quad+\sum_{i=2}^{n} f\left(y_{1}, x_{2}, \ldots, d\left(x_{i}\right), \ldots, x_{n}\right), f\left(x_{1}, \ldots, x_{n}\right)\right]_{2 k} \\
& \quad+\sum_{j=0}^{2 k-1}\left[\left[\left[f\left(y_{1}, x_{2}, \ldots, x_{n}\right), f\left(x_{1}, \ldots, x_{n}\right)\right]_{j}, d\left(f\left(x_{1}, \ldots, x_{n}\right)\right)\right],\right. \\
& \left.f\left(x_{1}, \ldots, x_{n}\right)\right]_{2 k-j-1}=0 .
\end{aligned}
$$

The second sum vanishes since, for each $j$,

$$
\begin{gathered}
{\left[\left[\left[f\left(y_{1}, x_{2}, \ldots, x_{n}\right), f\left(x_{1}, \ldots, x_{n}\right)\right]_{j}, d\left(f\left(x_{1}, \ldots, x_{n}\right)\right)\right], f\left(x_{1}, \ldots, x_{n}\right)\right]_{2 k-j-1}} \\
=\sum_{i=0}^{2 k-j-1}\left(\begin{array}{c}
2 k-j-1 \\
i
\end{array}\right)\left[\left[f\left(y_{1}, x_{2}, \ldots, x_{n}\right), f\left(x_{1}, \ldots, x_{n}\right)\right]_{j+i}\right. \\
\left.\left[d\left(f\left(x_{1}, \ldots, x_{n}\right)\right), f\left(x_{1}, \ldots, x_{n}\right)\right]_{2 k-j-i-1}\right]=0
\end{gathered}
$$

where either $j+i \geq k$ or $2 k-j-i-1 \geq k$. Thus we have

$$
\begin{aligned}
& {\left[f^{d}\left(y_{1}, x_{2}, \ldots, x_{n}\right)+f\left(d\left(y_{1}\right), x_{2}, \ldots, x_{n}\right)\right.} \\
& \left.\quad+\sum_{i=2}^{n} f\left(y_{1}, x_{2}, \ldots, d\left(x_{i}\right), \ldots, x_{n}\right), f\left(x_{1}, \ldots, x_{n}\right)\right]_{2 k}=0 .
\end{aligned}
$$

Again by Kharchenko's theorem, we have

$$
\begin{aligned}
& {\left[f^{d}\left(y_{1}, x_{2}, \ldots, x_{n}\right)+f\left(z, x_{2}, \ldots, x_{n}\right)\right.} \\
& \left.\quad+f\left(y_{1}, y_{2}, x_{3}, \ldots, x_{n}\right)+\cdots+f\left(y_{1}, x_{2}, \ldots, y_{n}\right), f\left(x_{1}, \ldots, x_{n}\right)\right]_{2 k}=0
\end{aligned}
$$

for all $x_{1}, \ldots, x_{n}, y_{1}, \ldots, y_{n}, z \in R$. In particular,

$$
\left[f^{d}\left(y_{1}, x_{2}, \ldots, x_{n}\right), f\left(x_{1}, \ldots, x_{n}\right)\right]_{2 k}=0
$$

and

$$
\left[f\left(y_{1}, y_{2}, x_{3}, \ldots, x_{n}\right), f\left(x_{1}, \ldots, x_{n}\right)\right]_{2 k}=0
$$

for all $x_{1}, \ldots, x_{n}, y_{1}, y_{2} \in R$. Continuing this process, we will finally get

$$
\left[f\left(y_{1}, \ldots, y_{n}\right), f\left(x_{1}, \ldots, x_{n}\right)\right]_{n k}=0
$$

for all $x_{1}, \ldots, x_{n}, y_{1}, \ldots, y_{n} \in R$. In light of the inner case, we have $f\left(y_{1}, \ldots, y_{n}\right) \in$ $\mathcal{Z}$ for all $y_{1}, \ldots, y_{n} \in R$, that is, $f\left(X_{1}, \ldots, X_{n}\right)$ is central-valued on $R$, except when char $R=2$ and $R$ satisfies $s_{4}$.

\section{REFERENCES}

1. C. L. Chuang, GPIs having coefficients in Utumi quotient rings, Proc. Amer. Math. Soc. 103 (1988), 723-728. MR 89e:16028

2. T. S. Erickson, W. S. Martindale and J. M. Osborn, Prime nonassociative algebras, Pacific J. Math. 60 (1975), 49-63. MR 52:3264

3. N. Jacobson, Structure of Rings, Amer. Math. Soc. Colloq. Pub., 37, Amer. Math. Soc., Providence, RI, 1964. MR 36:5158 
4. V. K. Kharchenko, Differential identities of semiprime rings, Algebra and Logic 18 (1979), 58-80. MR 81f:16052

5. C. Lanski, Differential identities, Lie ideals, and Posner's theorems, Pacific J. Math. 134 (1988), 275-297. MR 89j:16051

6. C. Lanski, An Engel condition with derivation, Proc. Amer. Math. Soc. 118 (1993), 731-734. MR 93i: 16050

7. P. H. Lee and T. K. Lee, Lie ideals of prime rings with derivations, Bull. Inst. Math. Acad. Sinica 11 (1983), 75-80. MR 85c:16049

8. U. Leron, Nil and power-central polynomials in rings, Trans. Amer. Math. Soc. 202 (1975), 97-103. MR 50:7241

9. W. S. Martindale, Prime rings satisfying a generalized polynomial identity, J. Algebra 12 (1969), 576-584. MR 39:257

10. E. C. Posner, Derivations in prime rings, Proc. Amer. Math. Soc. 8 (1957), 1093-1100. MR 20:2361

11. J. Vukman, Commuting and centralizing mappings in prime rings, Proc. Amer. Math. Soc. 109 (1990), 47-52. MR 90h:16010

Department of Mathematics, National Taiwan University, Taipei, Taiwan

E-mail address: phlee@math.ntu.edu.tw

Department of Mathematics, National Taiwan University, Taipei, Taiwan

E-mail address: tklee@math.ntu.edu.tw 